\title{
Band offsets and trap-related electron transitions at interfaces of (100)InAs with atomic-layer deposited $\mathrm{Al}_{2} \mathrm{O}_{3}$
}

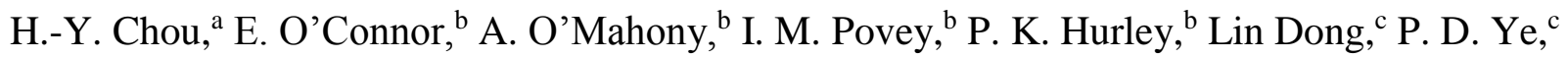
V. V. Afanas'ev, ${ }^{a}$ M. Houssa, ${ }^{a}$ A. Stesmans ${ }^{a}$

${ }^{a}$ Laboratory of Semiconductor Physics, Department of Physics, University of Leuven, Belgium

${ }^{\mathrm{b}}$ Tyndall National Institute, University College Cork, Lee Maltings, Prospect Row, Cork, Ireland ${ }^{\mathrm{c} S}$ School of Electrical and Computer Engineering and Birck Nanotechnology Center, Purdue University, West Lafayette, Indiana 47907, USA

\begin{abstract}
In order to develop methodology enabling characterization of electron states at semiconductor/insulator interfaces containing an interlayer we addressed spectroscopy of photocurrents in (100)InAs/ $\mathrm{Al}_{2} \mathrm{O}_{3} /$ metal structures which is shown to contain two contributions: One related to the internal photoemission (IPE) of electrons from the electrodes into alumina and another stemming from trapping-related displacement currents. Analysis of the IPE spectra suggests that the out-diffusion of In and, possibly, its incorporation in $\mathrm{Al}_{2} \mathrm{O}_{3}$, leads to the development of $\approx 0.4 \mathrm{eV}$ wide conduction band (CB) tail states. At the same time, the top of the InAs valence band measured relative to the alumina $\mathrm{CB}$ bottom remains at the same energy value of $3.45 \pm 0.10 \mathrm{eV}-$ as also found for the earlier studied case of $\mathrm{GaAs} / \mathrm{Al}_{2} \mathrm{O}_{3}$ - supporting the validity of the "common anion" rule. However, atomic-layer deposition of alumina on InAs is found to result in additional low-energy electron transitions with spectral thresholds in the range of 2.0-2.2 eV, which is close to the bandgap of AlAs. This observation suggests interaction at the interface of As with Al, resulting in the formation of an interlayer with significant amount of Al-As bonds that effectively lower the barrier for electrons.
\end{abstract}




\section{Introduction}

Application of insulating metal oxides to high-mobility semiconductors (Ge and related $\mathrm{SiGe}$, GeSn alloys, group $\mathrm{A}_{\mathrm{III}} \mathrm{B}_{\mathrm{V}}$ materials, etc.) usually results in interfaces with by far more complex electronic structure than that of more conventional silicon/oxide systems [1]. This is primarily related to the exposure of the semiconductor surface to oxidant during insulator deposition, leading to the growth of a "native" oxide interlayer (IL). In contrast with the silicon case where a wide-bandgap $\mathrm{SiO}_{2}$ IL is formed, oxidation of high-mobility semiconductors typically leads to compounds with a more narrow bandgap $\left(\mathrm{In}_{2} \mathrm{O}_{3}, \mathrm{Ga}_{2} \mathrm{O}_{3}, \mathrm{GeO}_{\mathrm{x}}\right)$ [2]. Furthermore, the oxides in the IL are often substoichiometric and may host gap electron states that enable trap-assisted tunneling and effectively lower interface barriers. In some cases even segregation of one element, e.g., arsenic [3,4], at the interface is encountered as well as in-diffusion of semiconductor atoms (Ge, Sn, In) into the insulating oxide layer [5,6]. Evaluation of the effect these factors have on the interface barriers represents a significant experimental challenge because transport of charge carriers involves not only intrinsic band states of the semiconductor and insulator materials, but also the IL-related contributions which are to be isolated on the background of intrinsic bands. In this work we will demonstrate the possibility to solve this problem by means of photocurrent spectroscopy, enabling separation between the internal photoemission (IPE) and the displacement currents related to trap-related transitions at the semiconductor/insulator interface.

Besides dealing with the interface characterization methodology, the present study addresses interfaces of indium arsenide (InAs) with $\mathrm{Al}_{2} \mathrm{O}_{3}$, of much interest by itself since in recent years the InAs semiconductor has been proposed as a candidate material for a wide spectrum of electronic and optoelectronic devices. For example, atomic-layer deposition (ALD) of $\mathrm{Al}_{2} \mathrm{O}_{3}$ is suggested as a formation of a passivation layer allowing one to reduce leakage current in InAs/GaSb super-lattice 
photodetectors [7]. Similarly, the $\mathrm{InAs} / \mathrm{Al}_{2} \mathrm{O}_{3}$ stack can be used to improve the electrical quality of GaSb p-type channels [8-10]. Most importantly, InAs is considered as a high mobility electron channel in a variety of transistor configurations ranging from planar metal-oxide-semiconductor (MOS) fieldeffect transistors (FETs) [11-13] to nano-wire FETs [14,15] and band-to-band tunneling devices $[16,17]$. The major difficulty in realization of practically useful InAs-based transistors appears to be the poor electrical quality of interfaces with deposited oxide insulators. A high density of charge traps generally encountered at the InAs/oxide interfaces leads to degradation of electron mobility, increases the sub-threshold slope, enhances noise, etc. [18,19]. Most of the effects correlate with electron trapping in the near-interface oxide layer(s) which brings up the above mentioned concern regarding the height of the energy barriers electrons encounter at the interfaces of InAs with oxide insulators. In particular, the role of InAs oxidation during insulator deposition leading to the formation of an IL remains unclear, since the high diffusivity [6] of In can drastically modify the IL composition, e.g., making it very different from that found at earlier studied GaAs interfaces. Furthermore, diffusion of highly mobile In and its incorporation into the atomic matrix of the insulating oxide itself may significantly affect the electronic properties of the dielectric as suggested by observations of annealing-induced variations in the band alignment at the interfaces of $\operatorname{In}_{0.53} \mathrm{Ga} 0.47 \mathrm{As}$ with insulating $\mathrm{Al}_{2} \mathrm{O}_{3}[20]$.

Moreover, there is significant inconsistency between the $\mathrm{InAs} / \mathrm{Al}_{2} \mathrm{O}_{3}$ band offsets reported in the literature: The results previously inferred from the heterojunction measurements, relying on the band offset transitivity hypothesis, suggest that the valence bands (VBs) of GaAs and InAs are energetically aligned, i.e., they follow the so-called "common anion rule" (cf. Fig. 1 in Ref. 21). However, the compilation based on the electron affinity values points to $\mathrm{a} \approx 0.25 \mathrm{eV}$ upshift of the VB top in $\operatorname{In}_{\mathrm{x}} \mathrm{Ga}_{1}$ $x$ As already for $x \approx 0.5$ (cf. Fig. 18 in Ref. 22). The latter prediction appears inconsistent with the IPE 
results indicating that the energy barrier, $\Phi_{\mathrm{e}}$, between the VB top in $\operatorname{In}_{\mathrm{x}} \mathrm{Ga}_{1-\mathrm{x}} \mathrm{As}(0 \leq \mathrm{x} \leq 0.53)$ alloys and the bottom of the oxide conduction band $(\mathrm{CB})$ remains constant at the interfaces with $\mathrm{HfO}_{2}\left(\Phi_{\mathrm{e}}=3.35\right.$ $\mathrm{eV})$ and $\mathrm{Al}_{2} \mathrm{O}_{3}\left(\Phi_{\mathrm{e}}=3.45 \mathrm{eV}\right)[23]$, in line with the results for the single-crystal (100) $\mathrm{InAs} / \mathrm{Al}_{2} \mathrm{O}_{3}$ interfaces $\left(\Phi_{\mathrm{e}}=3.45 \mathrm{eV}\right.$, Ref. 24). However, a somewhat lower energy barrier between $\operatorname{In}_{\mathrm{x}} \mathrm{Ga}_{1-\mathrm{x}} \mathrm{As} \mathrm{VB}$ and the bottom of the $\mathrm{Al}_{2} \mathrm{O}_{3} \mathrm{CB}, \Phi_{\mathrm{e}}=3.3 \mathrm{eV}$, has been reported for $\operatorname{In}_{\mathrm{x}} \mathrm{Ga}_{1-\mathrm{x}} \mathrm{As}(\mathrm{x}=0.53$ and $\mathrm{x}=0.75)$, which is further reduced upon annealing [20]. In the case of interfaces between thin epitaxial $\mathrm{In}_{0.53} \mathrm{Ga}_{0.47} \mathrm{As}$ and InAs layers with $\mathrm{Al}_{2} \mathrm{O}_{3}$, an even lower barrier of $\Phi_{\mathrm{e}}=3.2 \mathrm{eV}$ has been reported [25], though a later analysis of the $\mathrm{InAs} / \mathrm{Al}_{2} \mathrm{O}_{3}$ interfaces [26] seems to affirm the value $\Phi_{\mathrm{e}}=3.45 \mathrm{eV}$ from Ref. 24. The exposed barrier variability points to the possible impact of indium oxidation and/or diffusion on the band alignment. For example, the slope of the Schottky plots of the electron barrier $\Phi_{\mathrm{e}}$ shown in Refs. 26,27 would correspond to an unrealistically low value of the image force constant $\varepsilon_{\mathrm{i}}<1\left(\varepsilon_{\mathrm{i}}=1\right.$ in vacuum) indicating that the used assumption of an abrupt InAs/ $\mathrm{Al}_{2} \mathrm{O}_{3}$ (or $\mathrm{In}_{0.53} \mathrm{Ga}_{0.47} \mathrm{As} /$ $\mathrm{Al}_{2} \mathrm{O}_{3}$ ) interface is inadequate.

In this work we will present systematic analysis of the IPE spectra at interfaces of single-crystal (100)InAs with $\mathrm{Al}_{2} \mathrm{O}_{3}$, leading to the demonstration that there are two different contributions to the photocurrent, i.e., band-to band and band-to-trap transitions. Besides providing a reliable determination of the intrinsic band alignment at the InAs $/ \mathrm{Al}_{2} \mathrm{O}_{3}$ interface, the analysis indicates two significant effects, namely the formation of an IL with energy gap close to that of AlAs, and development of $\mathrm{CB}$ tail states in the $\mathrm{Al}_{2} \mathrm{O}_{3}$ layer which may be associated with in-diffusion of In during ALD of alumina. Eventually, the IL formation represents the critical factor impairing insulating properties of the oxide insulation. 


\section{Experimental}

Since revealing the effect of the oxidation-grown IL on the band alignment at the InAs/ $\mathrm{Al}_{2} \mathrm{O}_{3}$ interface represents one of the major goals of this study, we analyzed two types of samples fabricated by ALD of amorphous (a-) alumina $\left[\mathrm{Al}\left(\mathrm{CH}_{3}\right)_{3}(\mathrm{TMA})+\mathrm{H}_{2} \mathrm{O}, 250{ }^{\circ} \mathrm{C}\right.$, TMA pulse first $]$ on top of (100)-oriented single-crystal InAs wafers: One set of samples was prepared without removal of the native oxide prior to the ALD and another one subjected to native oxide removal (denoted as "precleaned" samples) using a buffered oxide etchant (BOE: 6 pts. $40 \% \mathrm{NH}_{4} \mathrm{~F}+1$ pt. $49 \% \mathrm{HF}$ mixture), i.e., 80 s etching in 1:5 $\mathrm{H}_{2} \mathrm{O}: \mathrm{BOE}$ solution. The substrates used were (100)InAs single-crystals of $\mathrm{n}$ - and ptype conductivity with dopant concentrations of $\approx 4 \times 10^{17}$ and $\approx 2 \times 10^{17} \mathrm{~cm}^{-3}$, respectively. The thickness of the insulating a- $\mathrm{Al}_{2} \mathrm{O}_{3}$ layers was 8 or $20 \mathrm{~nm}$. For the sake of comparison, similar alumina layers were deposited by ALD on top of imec-cleaned [28] (100) silicon wafers. MOS capacitors were fabricated by deposition of semitransparent (13-nm thick) top metal (Au or $\mathrm{Al}$ ) electrodes of $0.5 \mathrm{~mm}^{2}$ area, while a $0.5-\mu \mathrm{m}$ thick Al blanket electrode was used as the backside contact. In both cases, the metallization was done by thermoresistive evaporation of the metal on an unheated substrate in high vacuum to avoid radiation damage of the sample.

The fabricated capacitors were used in IPE and photoconductivity (PC) experiments conducted at room temperature over the spectral range 1.9-6.5 eV with a constant spectral resolution of $2 \mathrm{~nm}$. As described earlier [24,29], the quantum yield (Y) is defined as the photocurrent normalized to the incident photon flux. The spectral dependences of the yield were measured under different bias voltages applied to the top metal electrodes and then analyzed to find spectral thresholds of different charge injection processes. By comparing the photocurrent yield spectra measured in capacitors with different metal gate material and/or under different orientation of the electric field in alumina layer, the injecting interface and, therefore, type of the photoinjected charge carrier can be identified [30]. As 
compared to the previous studies [24,29], extensive signal averaging (>100) was applied to enable reliable detection of low-level displacement currents with the sensitivity in the $10^{-17} \mathrm{~A}$ range.

\section{Results and Discussion}

Figure 1 shows an example of the photocurrent yield spectra corresponding to electron IPE from the InAs substrate (positive top metal bias) as measured on samples prepared by ALD of alumina on InAs substrates with native oxide on top and on substrate subjected to the BOE surface clean. The absence of a substantial influence of the native oxide etching indicates that during ALD, the oxidized As and In compounds are effectively eliminated by the TMA, - a well known "self-cleaning" process [31-33]. Two features are observed in all spectral curves at $h v=4.4 \mathrm{eV}$ and $4.6 \mathrm{eV}$, marked by vertical arrows in Fig. 1. These energies match the known energies of optical singularities associated with excitation of direct transitions $\left(\mathrm{E}_{0}{ }^{\prime}, \mathrm{E}_{0}{ }^{\prime}+\Delta_{0}{ }^{\prime}\right)$ and $\mathrm{E}_{2}$ in the InAs crystal [34-36], respectively. This observation indicates that the photocurrent across the insulating alumina layer originates from electron IPE from the InAs substrate. However, no optical features corresponding to $E_{1}$ and $E_{1}+\Delta_{1}$ singularities can be seen in the spectral range $h v=2.4-2.8 \mathrm{eV}$ which does suggest that the photocurrent generation mechanism at lower photon energies is unrelated to optical excitations of electrons in InAs. Rather, the featureless spectral curves in the range $h v=2.0-3.5 \mathrm{eV}$ resemble the signals related to the excitation of electron states inside the IL between InAs and the oxide on top [37]. Therefore, the yield spectra shown in Fig. 1 apparently contain contributions stemming from at least two different photocurrent generation mechanisms.

The latter conclusion is supported by the analysis of the yield spectra measured under negative top metal bias on $n$ - and p-type (100)InAs/ $/ \mathrm{Al}_{2} \mathrm{O}_{3} / \mathrm{Au}$ samples and shown in panels (a) of Figs. 2 and 3, 
respectively. The reversal of the electric field splits the IPE spectra in two clearly distinctive parts: At $h v>3.5 \mathrm{eV}$, the photocurrent flow corresponds to electron drift from the top metal electrode towards the InAs substrate and can be associated with electron IPE from Au. However, at lower photon energies, a featureless spectrum of photocurrent of opposite direction is observed, with spectral appearance closely resembling an attenuated signal $(\approx 10$ times $)$ seen in the same spectral range under positive bias (cf. Fig. 1). Obviously, electrons injected from InAs cannot drift across the $\mathrm{Al}_{2} \mathrm{O}_{3}$ layer against the electric field of repulsive polarity. Thus, the low-energy signal probably originates from a displacement current caused by recharging of traps in the insulator near the interface of InAs with $\mathrm{Al}_{2} \mathrm{O}_{3}$. Indeed, the ALD-grown alumina layers are known to contain a considerable density of electron traps (acceptor states) [38,39] which may trap electrons optically excited in the nearby electrode leading to the observed low-level (<10 fA) re-charging current.

In order to verify the hypothesis regarding the trap-related current, photocurrent yield spectra were also measured on samples with Al metal electrodes instead of Au. The corresponding spectral curves are shown in panels (b) of Figs. 2 and 3 for the n- and p-type InAs/ $\mathrm{Al}_{2} \mathrm{O}_{3} / \mathrm{Al}$ capacitors, respectively. As expected, due to the lower work function of $\mathrm{Al}$ as compared to $\mathrm{Au}$, the energy onset of electron IPE from the negatively biased top metal electrode is shifted to the lower photon energy $\Phi_{\mathrm{e}}(\mathrm{Al})[\mathrm{cf}$. Fowler plot shown in the inset in Fig. 3(b)]. But what is more revealing is that in the samples with Al metallization the low-energy photocurrent $[\mathrm{h} v<3.5 \mathrm{eV}$ in Fig. 2(b)] measured under positive metal bias changes the direction to the opposite, corresponding to electron motion from the Al gate towards the InAs substrate. This result allows us to associate this current with the capturing of electrons optically excited in the $\mathrm{Al}$ electrode by traps in the near-interface alumina layer. From the ratio between the photocurrent yield measured under negative bias and that observed under the reversed field orientation $\left[\approx 10^{2}\right.$, cf. Fig. $\left.2(\mathrm{~b})\right]$, it becomes even possible to evaluate the average displacement of electrons in the 
direction opposite to the field as $\approx 1 \%$ of the alumina thickness, i.e., $\approx 0.2 \mathrm{~nm}$. This length reflects the mean free path of an electron during its ballistic transport from $\mathrm{Al}$ into $\mathrm{Al}_{2} \mathrm{O}_{3}$. In turn, the optical excitation of electrons inside the IL between InAs and $\mathrm{Al}_{2} \mathrm{O}_{3}$ followed by their trapping in alumina would explain the low energy photocurrents in the Au-gated InAs/ $\mathrm{Al}_{2} \mathrm{O}_{3} /$ metal entities discussed in the previous paragraph.

An important result of the analysis of the Al-metallized samples consists in the clear separation between the spectral ranges corresponding to the trap-related displacement currents and to the signal caused by electron IPE from InAs into $\mathrm{Al}_{2} \mathrm{O}_{3}$ : As one can see from the spectra shown in panels (b) in Figs. 2 and 3 for the case of positive Al gate biasing, with increasing photon energy, the IPE current takes over the trap-related signal at $\mathrm{h} v>3.5 \mathrm{eV}$ and, therefore, this high-energy part of the IPE spectral curves can be used to reliably determine the IPE spectral threshold. This conclusion is independently supported by the observed stronger field dependence of the IPE threshold in the p-type $\mathrm{InAs} / \mathrm{Al}_{2} \mathrm{O}_{3} / \mathrm{Al}$ sample [Fig. 3(b)] compared to the n-InAs case [Fig. 2(b)]. Indeed, this field effect is consistent with the apparent barrier lowering due to penetration of electric field into the depleted p-InAs layer [24,30] causing an additional shift of the spectral threshold by a value comparable to the InAs bandgap width $(0.36 \mathrm{eV}$ at $300 \mathrm{~K})$.

To find the spectral threshold of electron IPE from the VB of InAs, the yield spectra measured under positive metal bias were re-plotted using the Powell coordinates, $\mathrm{Y}^{1 / 3}-\mathrm{h} v,[40]$ as illustrated in Fig. 4 for the n-type (100)InAs/a- $\mathrm{Al}_{2} \mathrm{O}_{3}(20 \mathrm{~nm}) / \mathrm{Au}$ sample. The observed abrupt increase of the yield above the level of the sub-threshold displacement current indicates the onset of electron photoemission from the InAs substrate. However, while for low bias voltages one can easily find the spectral threshold labeled as $\Phi_{\mathrm{e}}($ high), with increasing strength of the electric field in the alumina the threshold 
splits, suggesting the presence of an additional field-activated electron injection mechanism characterized by a somewhat lower energy threshold $\Phi^{*}{ }_{\mathrm{e}}(\mathrm{high})$. The latter effect is probably related to the band-tail states below the CB bottom edge in amorphous alumina induced by In doping: A similar $\approx 0.3-0.4 \mathrm{eV}$ barrier lowering is observed at the $\mathrm{Al} / \mathrm{Al}_{2} \mathrm{O}_{3}$ and $\mathrm{Au} / \mathrm{Al}_{2} \mathrm{O}_{3}$ interfaces in the samples grown on (100)InAs substrates as compared to their counterparts fabricated on (100)Si. This is illustrated by the semi-logarithmic plot of the IPE spectra and the Fowler $\left(\mathrm{Y}^{1 / 2}-\mathrm{h} v\right)$ plots shown in Figs. 5 (a) and (b), respectively. It is also worth adding that $\mathrm{Al}_{2} \mathrm{O}_{3}$ layers grown on InAs exhibit an enhanced sub-threshold photoconductivity in the photon energy range near and below $\mathrm{Eg}_{\mathrm{g}}\left(\mathrm{a}-\mathrm{Al}_{2} \mathrm{O}_{3}\right) \approx 6$ eV compared to the samples grown on $\mathrm{Si}$. The latter is also illustrated in the inset in Fig. 5(a), which compares the PC yield spectra of 20-nm thick alumina layers grown by ALD on top of two different semiconductors, i.e., InAs and $\mathrm{Si}$. The effect of indium in-diffusion would also explain earlier observations regarding barrier height lowering caused by high-temperature annealing of the samples prepared by $\mathrm{ALD}$ of $\mathrm{Al}_{2} \mathrm{O}_{3}$ on In-containing semiconductor substrates [20].

To determine the intrinsic (zero-field) barrier height between the InAs $\mathrm{VB}$ and the $\mathrm{Al}_{2} \mathrm{O}_{3} \mathrm{CB}$ the inferred IPE spectral thresholds, $\Phi_{\mathrm{e}}($ high), are plotted using the Schottky coordinates as illustrated in Fig. 6(a). Linear extrapolation to zero field value yields the barriers heights in the range 3.45-3.50 $\mathrm{eV}$ which coincide [within the accuracy of the measurements $(0.05-0.1 \mathrm{eV})]$ with the earlier reported barriers at interfaces of GaAs and $\operatorname{In}_{x} \mathrm{Ga}_{1-\mathrm{x}} \mathrm{As}(\mathrm{x} \leq 0.53)$ alloys with ALD alumina grown on top [23]. In turn, the somewhat lower values of the barrier of 3.2-3.3 eV reported in the literature $[20,25]$ can be explained by the electron photoinjection via the alumina CB tail states characterized by the lower threshold $\Phi^{*}{ }_{\mathrm{e}}(\mathrm{high})$. Therefore, we may conclude that replacement of Ga by In in arsenide semiconductors does not change the energy of the VB top, i.e., the bandgap narrowing from $1.42 \mathrm{eV}$ in GaAs to $0.36 \mathrm{eV}$ in InAs occurs predominantly by the shift of the CB bottom edge. The same energy 
of the VB top in the studied arsenides suggests validity of the so-called "common anion rule" which associates the VB states with common anions in the compounds, in the studied case - group V atoms.

For the sake of comparison, in Fig. 6(a) are also shown the values -falling in the range 2.1-2.3 $\mathrm{eV}$ - of the low-energy threshold $\Phi_{\mathrm{e}}(\mathrm{low})$ inferred from the trap-related current spectra, that are associated with electron excitation in the $\mathrm{IL}$ formed between InAs and $\mathrm{Al}_{2} \mathrm{O}_{3}$. Worth of noticing here is that the such spectral thresholds, in the 2.0-2.2 eV energy range have also been reported in the literature for interfaces of different In-containing semiconductors, including not only InAs [25,26] but also $\operatorname{In}_{\mathrm{x}} \mathrm{Ga}_{1-\mathrm{x}} \mathrm{As}[20,27]$ with the ALD-grown alumina. The universal appearance of this threshold points to a similar origin of the electron states in these ILs. Namely, the threshold at about $2.2 \mathrm{eV}$ is very close to the bandgap width of AlAs $(2.16 \mathrm{eV}$ at $300 \mathrm{~K})$. This observation may suggest that while In diffuses away from the interface region, the remaining As atoms interact with Al during the ALD of alumina. Indeed, on the basis of atomic analysis, it has even been suggested that bonding of As to Al may lead to the formation of a thin AlAs layer during ALD [32]. Though the available transmission electron microscopy (TEM) images [25,26] cannot directly support this hypothesis because of insufficient Z-contrast, the formation of Al-As bonds inside the IL and the corresponding electron states is well possible. It would then be logical to assign the low-energy $(\mathrm{h} v<3.5 \mathrm{eV}$ ) portion of the photocurrent yield spectra to optical excitation of AlAs-derived states in the IL followed by electron trapping in the near-interface alumina layer. Using this inference, the resulting In $\mathrm{As} / \mathrm{Al}_{2} \mathrm{O}_{3}$ interface band diagram can be schematically presented as shown in Fig. 6(b). If assuming that the upper edge of the occupied states in the AlAs IL is energetically also aligned with the top of the InAs VB, as it is shown to be the case in GaAs, the IL in the current case will provide the energetically lowest barrier, of around $2 \mathrm{eV}$, for electron injection from the semiconductor $\mathrm{VB}$ into $\mathrm{Al}_{2} \mathrm{O}_{3}$. This hypothesis may also explain the earlier result on the determination of the tunneling barrier between these materials 
revealing only $\approx 2.3-\mathrm{eV}$ barrier [11] which appears to be significantly lower than the $\approx 3.1 \mathrm{eV}$ fundamental $\mathrm{CB}$ offset between InAs and $\mathrm{Al}_{2} \mathrm{O}_{3}$. Obviously then, tight control of the IL is needed to ensure a low electron injection rate from InAs into the insulating oxide which may otherwise cause charge instability in the gate stack.

\section{Conclusions}

Our results indicate that the "standard" model of an abrupt semiconductor/insulator transition cannot be used to adequately describe the spectrum of electron states at interfaces of InAs with ALDgrown $\mathrm{Al}_{2} \mathrm{O}_{3}$ on top. Nevertheless, despite the more complex electron level structure, our photocurrent analysis has enabled the determination of the band alignment between InAs and $\mathrm{Al}_{2} \mathrm{O}_{3}$, which is found to be insensitive to the pre-deposition cleaning of the InAs surface and probably reflects intrinsic properties of the interface. The analysis of IPE spectra reveals two additional factors contributing to the complexity of the picture: First, the out-diffusion of In and, possibly, its incorporation into the insulating oxide, leads to the development of $\approx 0.4 \mathrm{eV}$ wide $\mathrm{CB}$ tail states. At the same time, the top of the InAs VB measured relative the alumina $\mathrm{CB}$ bottom remains at the same energy as in the earlier studied case of GaAs supporting the validity of the "common anion" rule. Therefore, the bandgap narrowing in the $\operatorname{In}_{\mathrm{x}} \mathrm{Ga}_{1-\mathrm{x}} \mathrm{As}$ semiconducting alloys with increasing In content should predominantly occur through the shift of the CB bottom edge. Second, interaction of the remaining As at the interface with Al during ALD apparently leads to formation of an IL containing significant amount of Al-As bonds which provide additional low-barrier electron injection channel. The presence of this narrowgap IL represents the major factor degrading the insulating properties of alumina ALD-grown on InAs.

Acknowledgement. The work at KU Leuven is supported by Fonds Wetenschappelijk Onderzoek - Vlaanderen (Project G.OCO5.13). 


\section{References}

1. See, e.g., Advanced Gate Stacks for High-Mobility Semiconductors, edited by A. Dimoulas, E. Gusev, P. C. McIntyre, M. Heyns (Springer, Berlin, 2007).

2. V. V. Afanas'ev and A. Stesmans, In: Physics and Technology of high-k Gate Dielectrics -VII, edited by S. Kar, M. Houssa, S. VanElshocht, D. Landheer (Electrochemical Society, Pennington, NJ, 2009), pp. 95-103.

3. M. Herrera, M. Chi, M. Bonds, N. D. Browning, J. N. Woolman, R. E. Kvaas, S. F. Harris, D. R. Rhiger, and C. J. Hill, Appl. Phys. Lett. 93, 093106 (2008).

4. F. S. Aguirre-Tostado, M. Milojevic, B. Lee, J. Kim., and R. M. Wallace, Appl. Phys. Lett. 93, 172907 (2008).

5. C. Merckling, X. Sun, Y. Shimura, A. Franquet, B. Vincent, S. Takeuchi, W. Vandervorst, O. Nakatsuka, S. Zaima, R. Loo, and M. Caymax, Appl. Phys. Lett. 98, 192110 (2011).

6. I. Krylov, R. Winter, D. Ritter, and M. Eizenberg, Appl. Phys. Lett. 104, 243504 (2014).

7. O. Salihoglu, J. Vac. Sci. Technol. B 32, 051201 (2014).

8. A. Greene, S. Madisetti, P. Nagaiah, M. Yakimov, V. Tokranov, R. Moore, and S. Oktyabrsky, Solid State Electron. 78, 56 (2012).

9. A. Greene, S. Madisetti, M. Yakimov, V. Tokranov, and S. Oktyabrsky, Intl. J. High Speed Electron. Systems 23, 1450015 (20142).

10. M. Yokoyama, H. Yokoyama, M. Takenaka, and S. Takagi, Appl. Phys. Lett. 106, 122902 (2015).

11. N. Li, E. S. Harmon, J. Hyland, D. B. Salzman, T. P. Ma, Y. Xuan, and P. D. Ye, Appl. Phys. Lett. 92, 143507 (2008). 
12. F. Xue, H. Zhao, Y.-T. Chen, Y. Wang, F. Zhou, and J. C. Lee, Appl. Phys. Lett. 98, 082106 (2011).

13. K. Takei, R. Kapadia, E. Pils, S. Krishna, and A. Javey, Appl. Phys. Lett. 102, 153513 (2013).

14. J. A. del Alamo, Nature 479, 317 (2011).

15. C. H. Wang et al., AIP Advances 7, 047108 (2014).

16. M. Houssa, E. Chagarov, and A. Kummel, MRS Bull. 34, 504 (2009).

17. A. Seabough and Q. Zhang, Proc. IEEE 98, 2095 (2010).

18. G. Astromskas, K. Storm, and L.-E. Wernersson, Appl. Phys. Lett. 98, 013501 (2011).

19. K.-M. Persson, B. G. Malm, and L.-E. Wernersson, Appl. Phys. Lett. 103, 033508 (2013).

20. N. V. Nguyen, M. Xu, O. A. Kirillov, P.D. Ye, C. Wang, K. Cheung, and J. S. Suehle, Appl. Phys. Lett. 96, 052107 (2010).

21. S. Tiwari and D. J. Frank, Appl. Phys. Lett. 60, 630 (1992).

22. C. I. Hinkle, E. M. Vogel, P. D. Ye, and R. M. Wallace, Curr. Opinion in Solid State and Mater. Sci. 15, 188 (2011).

23. V. V. Afanas'ev, A. Stesmans, G. Brammertz, A. Delabie, S. Sionke, A. O’Mahony, I. M. Povey, M. E. Pemble, E. O’Connor, P. K. Hurley, and S. B. Newcomb, Appl. Phys. Lett. 94, 202110 (2009).

24. V. V. Afanas'ev, Adv. Condens. Matter Phys. 301302 (2014).

25. Q. Zhang, R. Li, R. Yan, T. Kosel, H. G. Xing, A. C. Seabaugh, K. Xu, O. A. Kirillov, D. J. Gundlach, C. A. Richter, and N. V. Nguyen, Appl. Phys. Lett. 102, 012101 (2013).

26. W. Li, Q. Zhang, R. Bijesh, O. A. Kirillov, Y. Liang, I. Levin, L.-M. Peng, C. A. Richter, X. Liang, S. Datta, D. J. Gundlach, and N. V. Nguyen, Appl. Phys. Lett. 105, 213501 (2014). 
27. Q. Zhang, G. Zhou, H. G. Xing, A. C. Seabaugh, K. Xu, H. Sio, O. A. Kirillov, C. A. Richter, and N. V. Nguyen, Appl. Phys. Lett. 100, 102104 (2012).

28. M. Meuris, S. Verhaverbeke, P. W. Mertens, H. F. Schmidt, M. M. Heyns, M. Kubota, A. Philipossian, K. Dillenbeck, D. Graf, A. Schnegg, and R. de Blank, Microelectron Eng. 22, 21 (1993).

29. V. V. Afanas'ev and A. Stesmans, J. Appl. Phys. 102, 081301 (2007).

30. V. V. Afanas'ev, Internal Photoemission Spectroscopy (Elsevier, Oxford, 2014).

31. A. P. Kirk, M. Milojevic, J. Kim, and R. M. Wallace, Appl. Phys. Lett. 96, 202905 (2010).

32. R. Timm, A. Fian, M. Hjort, C. Thelander, E. Lind, J. N. Andersen, L.-E. Wernersson, and A. Mikkelsen, Appl. Phys. Lett 97, 132904 (2010).

33. D. M. Zhernokletov, P. Laukkanen, H. Dong, R. V. Galatage, B. Brennan, M. Yakimov, V.

Tokranov, J. Kim, S. Oktyabrsky, and R. M. Wallace, Appl. Phys. Lett. 102, 211601 (2013).

34. D. E. Aspnes and A. A. Studna, Phys. Rev. B 27, 985 (1983).

35. T. H. Ghong, T. J. Kim, J. W. Jung, Y. D. Kim, and D. E. Aspnes, J. Appl. Phys. 103, 073502 (2008)

36. T. J. Kim, J. J. Yoon, S. Y. Hwang, D. E. Aspnes, Y. D. Kim, H. J. Kim, Y. C. Chang, and J. D. Song, Appl. Phys. Lett. 95, 111902 (2009).

37. V. V. Afanas'ev, J. Appl. Phys. 113, 166101 (2013).

38. M. B. Zahid, D. R. Aguado, R. Degraeve, W. C. Wang, B. Govoreanu, M. Toledano-Luque, V. V. Afanas'ev, and J. Van Houdt, IEEE Trans. Electron. Dev. 57, 2907 (2010).

39. V. V. Afanas'ev, W. C. Wang, F. Cerbu, O. Madia, M. Houssa, A. Stesmans, ECS Trans. 64(8) 17 (2014).

40. R. J. Powell, J. Appl. Phys. 41, 2424 (1970). 


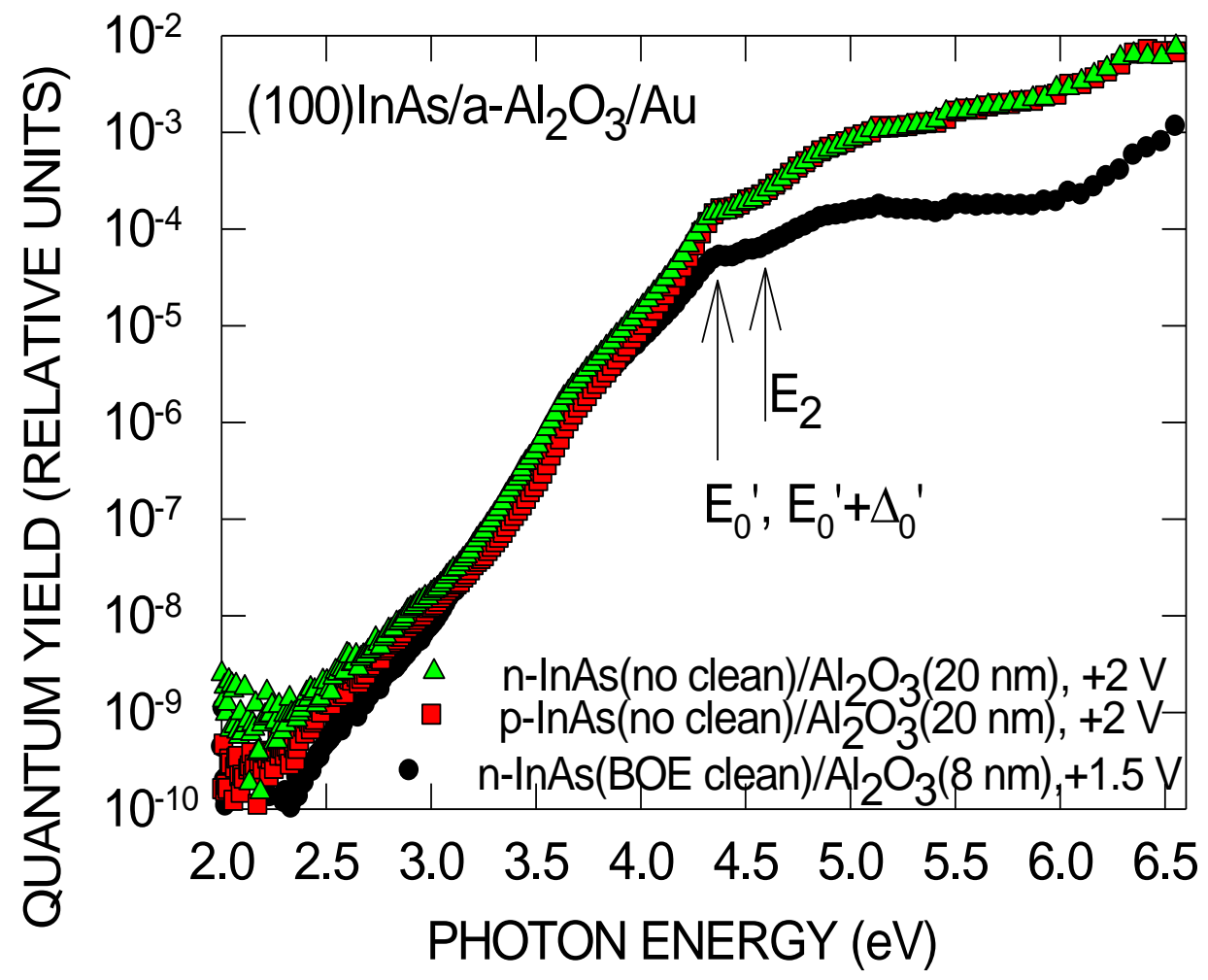

Fig. 1. Semi-logarithmic plot of the IPE quantum yield as a function of photon energy as measured on (100)InAs/a- $\mathrm{Al}_{2} \mathrm{O}_{3} / \mathrm{Au}$ samples prepared by using different InAs surface cleaning schemes. Vertical arrows $\mathrm{E}_{0}{ }_{0}, \mathrm{E}_{0}{ }_{0}+\Delta^{\prime}{ }_{0}$ and $\mathrm{E}_{2}$ mark the energies of direct optical transitions in the InAs crystal. 


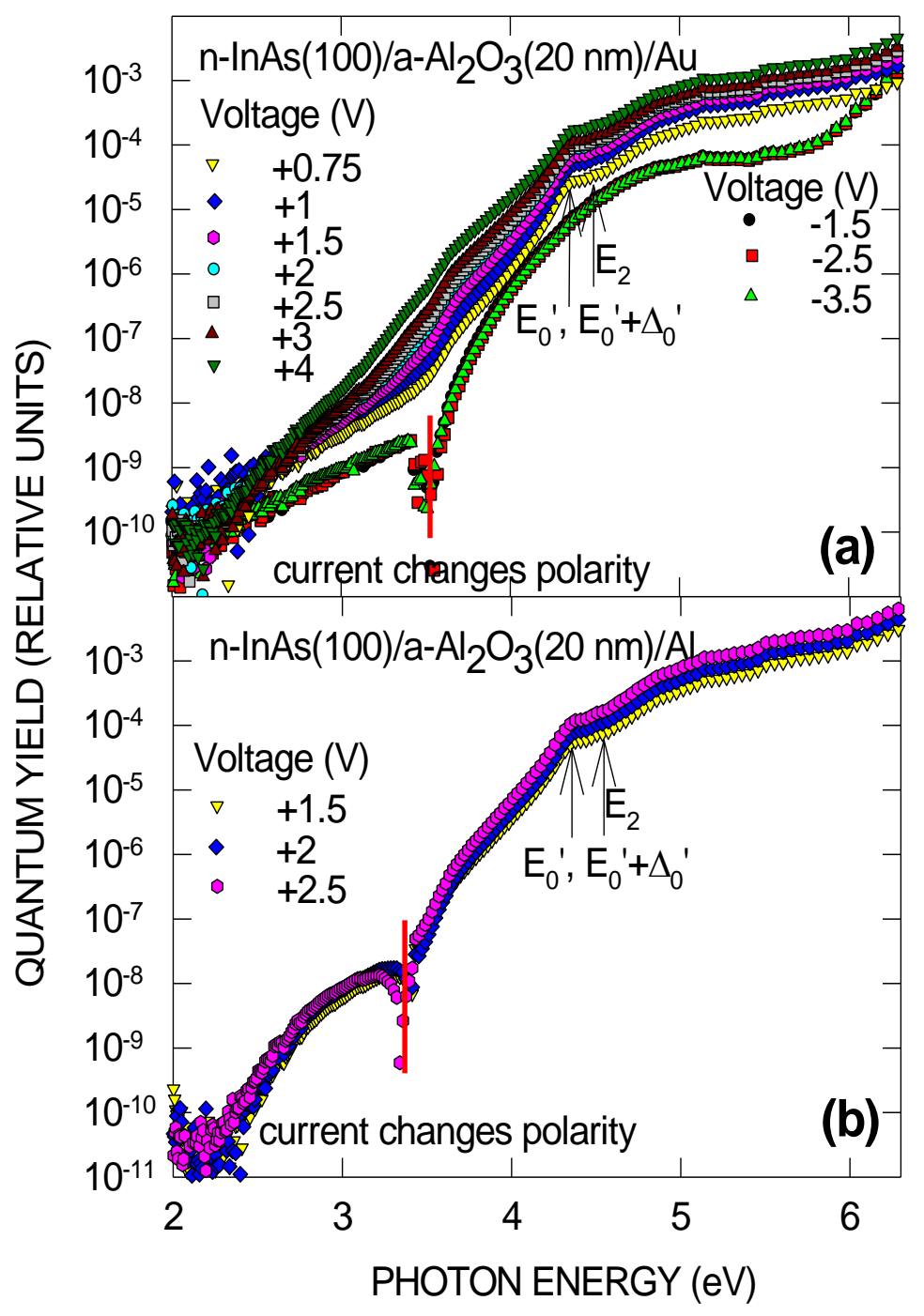

Fig. 2. Semi-logarithmic plot of the IPE quantum yield as a function of photon energy as measured on n-type (100)InAs/a- $\mathrm{Al}_{2} \mathrm{O}_{3}$ samples prepared on the un-etched InAs surface with $\mathrm{Au}$ (a) and $\mathrm{Al}$ (b) metal electrodes. The voltages applied to the metal are indicated in the legend. Vertical arrows E' ${ }_{0}$, $\mathrm{E}^{\prime}{ }_{0}+\Delta{ }^{\prime}{ }_{0}$ and $\mathrm{E}_{2}$ mark the energies of direct optical transitions in the InAs crystal. 


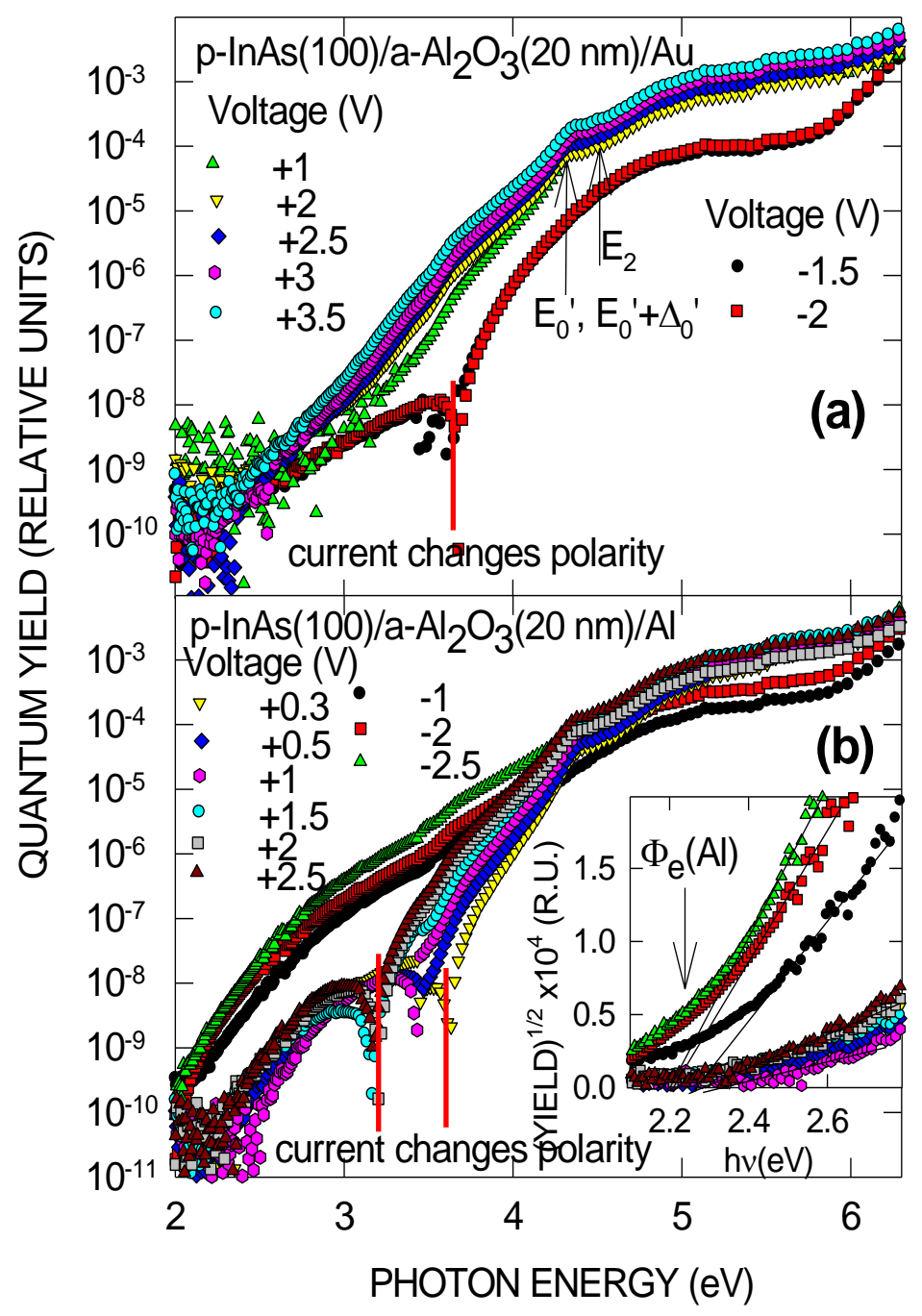

Fig. 3. Semi-logarithmic plot of the IPE quantum yield as a function of photon energy as measured on p-type (100)InAs/a- $\mathrm{Al}_{2} \mathrm{O}_{3}$ samples prepared on the un-etched InAs surface with $\mathrm{Au}$ (a) and $\mathrm{Al}$ (b) metal electrodes. The voltages applied to the metal are indicated in the legend. The vertical arrows E' 0 , $\mathrm{E}^{\prime}{ }_{0}+\Delta^{\prime}{ }_{0}$ and $\mathrm{E}_{2}$ mark the energies of direct optical transitions in the InAs crystal. The inset in panel (b) illustrates spectral threshold determination of electron IPE from $\mathrm{Al}$ into $\mathrm{Al}_{2} \mathrm{O}_{3} \Phi_{\mathrm{e}}(\mathrm{Al})$ using $\mathrm{Y}^{1 / 2}-\mathrm{h} v$ (Fowler) plots. 


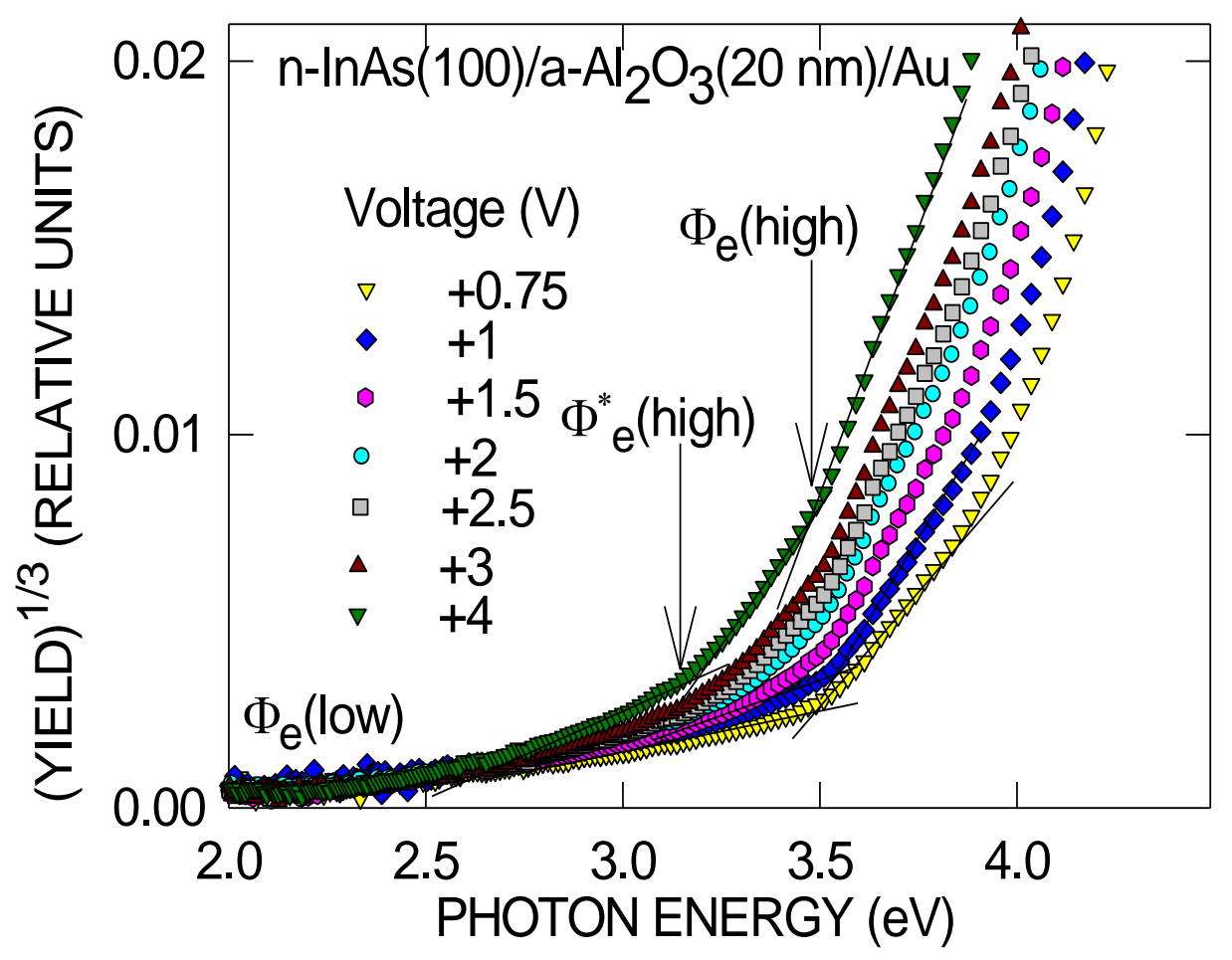

Fig. 4. Cube root of the IPE yield as a function of photon energy measured for different bias values applied to the n-type (100)InAs/a- $\mathrm{Al}_{2} \mathrm{O}_{3}(20 \mathrm{~nm}) / \mathrm{Au}$ capacitor, used to determine the spectral thresholds. The inferred thresholds $\Phi_{\mathrm{e}}$ (low/high) of electron IPE from the VB of InAs into the CB of $\mathrm{a}-\mathrm{Al}_{2} \mathrm{O}_{3}$ are indicated by arrows. Lines guide the eye. 

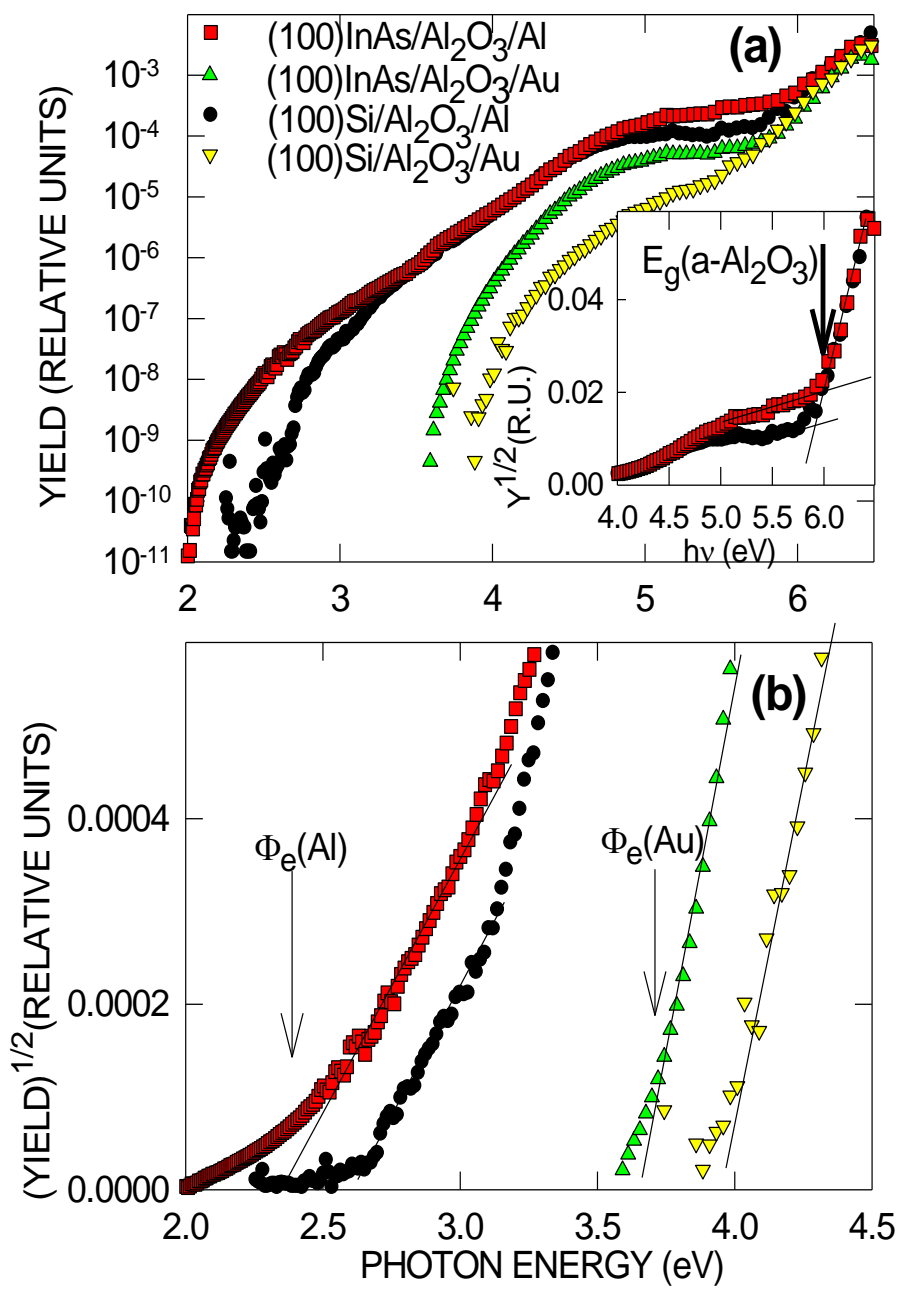

Fig. 5. Semi-logarithmic (a) and Fowler (b) plots of the IPE quantum yield as a function of photon energy as measured on p-type (100)InAs/a- $\mathrm{Al}_{2} \mathrm{O}_{3}$ and (100) $\mathrm{Si} / \mathrm{a}-\mathrm{Al}_{2} \mathrm{O}_{3}$ samples with $\mathrm{Au}$ and $\mathrm{Al}$ top electrodes under $-2 \mathrm{~V}$ bias voltage applied to the metal. The vertical arrows mark the threshold energies of electron IPE from the metal into the alumina layer. The inset in panel (a) shows the $\mathrm{Y}^{1 / 2}-\mathrm{h} v$ plot of the yield spectra in the photon energy range near the spectral threshold of the ALD alumina intrinsic photoconductivity. The vertical arrow marks the inferred bandgap of the alumina layer $\mathrm{Eg}_{\mathrm{g}}(\mathrm{a}-$ $\left.\mathrm{Al}_{2} \mathrm{O}_{3}\right)$. 

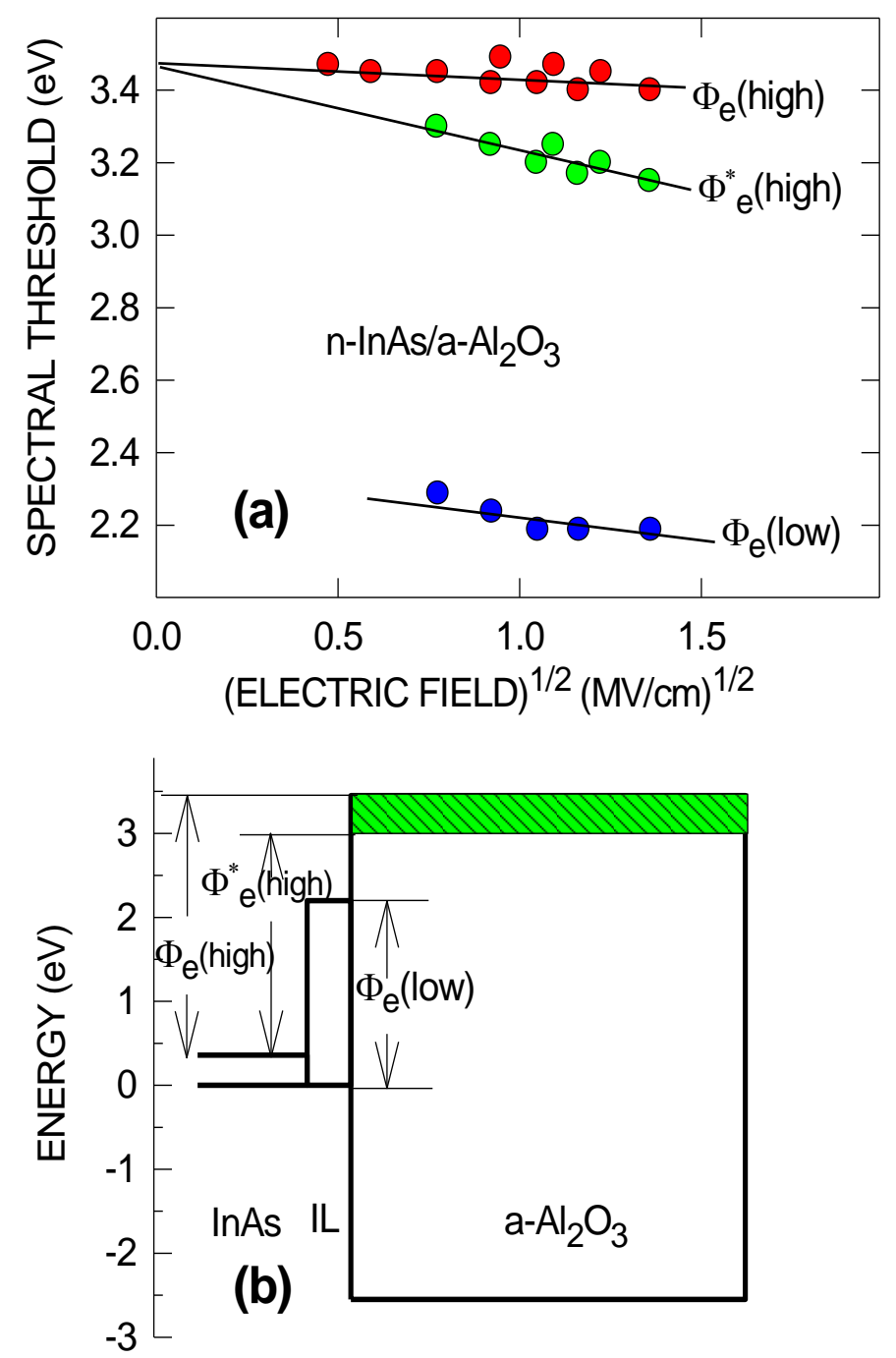

Fig. 6. (a) Determination of energy barrier at the InAs/a- $\mathrm{Al}_{2} \mathrm{O}_{3}$ interface using the Schottky plot of the IPE spectral thresholds; (b) Schematic zero-field energy band diagram of the InAs/a- $\mathrm{Al}_{2} \mathrm{O}_{3}$ interface derived from the current study. 